\title{
Análisis Morfométrico de la Mandíbula de Pacientes con Asimetría Facial Asociada a Hiperplasia Condilar. Estudio en Radiografía Panorámica
}

\author{
Morphometric Analysis of the Mandible in Patients with Facial Asymmetry \\ Associated to Condylar Hyperplasia. A Panoramic Radiography Study
}

\author{
Susana Zapata*; Hugo Medina*; Diego Saravia*; Pablo Navarro* \& Sergio Olate,***
}

\begin{abstract}
ZAPATA, S.; MEDINA, H.; SARAVIA, D.; NAVARRO, P. \& OLATE, S. Análisis morfométrico de la mandíbula de pacientes con asimetría facial asociada a hiperplasia condilar. Estudio en radiografía panorámica. Int. J. Morphol., 32(1):161-165, 2014.

RESUMEN: El objetivo de esta investigación es definir características morfológicas en radiografías panorámicas digitales de sujetos con deformidad facial asimétrica asociada a hiperplasia condilar. Se diseño un estudio de corte transversal para estudiar 14 pacientes con la patología identificándose puntos y lineas de medición de forma comparativa entre la hemimandíbula con diagnóstico de hiperplasia condilar ya la hemimandíbula cuyo condilo presenta un crecimiento normal; todas las mediciones fueron realizadas de forma manual de acuerdo a protocolos previamente establecidos. Las variables de análisis fueron tamaño condilar, tamaño de rama mandibular y tamaño del cuerpo de mandíbula en relación al foramen mentual. El análisis de datos fue realizado mediante la prueba Chi-cuadrado considrando el valor de $\mathrm{p}<0,05$ para obtener diferencias estadísticamente significativas. Los resultados mostraron que existían diferencias significativas entre ambos lados de la mandíbula en cinco de las nueve variables utilizadas en esta investigación; sin embargo, a partir de la medición de ambas hemimandibulas se obtuvo diferencias que llegaban a valores de 2 mm o menos, lo cual puede ser clínicamente intrascendente a pesar de la significancia estadística. Se concluye que es necesario obtener nuevas mediciones en otras áreas o con otra metodología que sea capaz de presentar diferencias estadísticas y clínicas representativas para realizar el diagnóstico precoz de la hiperplasia condilar.
\end{abstract}

PALABRAS CLAVE: Asimetría facial; Radiografía panorámica; Hiperplasia condilar.

\section{INTRODUCCIÓN}

La asimetría facial es una condición biológica común en diferentes sujetos (Ferrario et al., 1994); sin embargo, en algunas personar puede responder a procesos patológicos frecuentemente asociados a las enfermedades condilares de la articulación temporo mandibular (Olate et al., 2012). Las condiciones clínicas de diagnóstico de esta patología ya han sido analizados previamente demostrando que cerca de un $30 \%$ de los sujetos con asimetrías faciales presentan también fenómenos de hiperplasia condilar (Olate et al., 2013a), lo cual se asocia a alteraciones de la función mandibular, dolores articulares y faciales, alteraciones dentarias y oclusales, entre otras.

Los análisis previos de pacientes con asimetría facial asociada a hiperplasia condilar han demostrado claras diferencias entre el lado que presenta la hiperplasia y el lado que no la presenta (Olate et al., 2013b). Lo interesante de estos análisis es que se han cuantificado y determinado algunas relaciones posibles de encontrar en estos sujetos, de forma que se pueda diagnosticar de forma precoz la patología condilar; sin embargo, estos estudios han sido desarrollados en tomografías computadorizadas limitando su empleo a quienes presentan este tipo de exámenes (Olate et al., 2013b).

Por otra parte, la radiografía panorámica es uno de los exámenes mas utilizados para realizar diagnósticos en dientes y maxilares (Dryland \& Ellis, 1990) y es de amplio uso en el análisis previo de tratamientos de ortodoncia y cirugía, siendo necesario establecer elementos de observación que orienten sobre diagnóstico precoz del proceso de hiperplasia condilar asociada a la asimetría facial.

\footnotetext{
* División de Cirugía Oral y Maxilofacial, Universidad de La Frontera, Temuco, Chile.

** Centro de Investigación en Ciencias Biomédicas, Universidad Autónoma de Chile, Temuco, Chile.
} 
El objetivo de esta investigación es determinar las características morfométricas mandibulares de sujetos con asimetría facial asociada a hiperplasia condilar mediante el análisis de radiografías panorámicas.

\section{MATERIAL Y MÉTODO}

Se diseño un estudio descriptivo observacional de corte transversal para el análisis de 14 pacientes. La muestra fue obtenida de los pacientes de entre 18 y 40 años, sin distinción de sexo, que consultaron por tratamiento quirúrgico de la asimetría facial en la División de Cirugía Oral y Maxilofacial de la Universidad de La Frontera, estableciendose un diagnostico clínico e imagenológico de asimetría facial para todos ellos. Solo fueron incluidos los sujetos que presentaran asimetría facial de componente transversal u horizontal asociado a hiperplasia condilar unilateral y fueron excluidos los pacientes con otras anomalías del desarrollo, pacientes con historia de cirugía o trauma facial o dentario, pacientes con reabsorción condilar diagnosticada y pacientes portadores de asimetría facial no asociada a hiperplasia condilar.
Previamente, todos los sujetos fueron evaluados mediante una anamnesis rigurosa para determinar la progresión de la asimetría facial y dentaria durante los 6 meses previos al diagnóstico, estudio con tomografia computadorizada cone beam y estudio com SPECT de acuerdo al protocolo indicado por Olate et al. (2013c). El análisis con radiografía panorámica digital también fue realizado siguiendo una metodología descrita previamente por Pozzer et al. (2009); la captura de esta imagen se realizo en el equipo Pax Zenith (Vatech, Korea, 2011) y fueron obtenidas por dos técnicos con experiencia en este tipo de exámenes. Todas las mediciones se realizaron tanto en la hemimandibula con diagnostico de hiperplasia condilar $(\mathrm{H}-$ $\mathrm{HC}$ ) y en la hemimandibula con crecimiento condilar normal (h-NHC).

Todas las mediciones fueron realizadas de acuerdo a lo observado en la Figura 1, utilizándose el esquema de variables presentado en la Tabla I. Todos los datos fueron registrados en milímetros $(\mathrm{mm})$ y el análisis de datos se realizo mediante la prueba Chi-Cuadrado considerando un valor de $p<0,05$ para definir relaciones estadísticamente significativas.

Tabla I. Variables utilizadas para analizar las radiografías panorámicas de pacientes con asimetría facial asociada a hiperplasia condilar.

\begin{tabular}{|c|c|c|}
\hline Código & Variable & Definición \\
\hline V1 & Atura de rama & $\begin{array}{l}\text { Distancia axial entre el punto mas inferior de la escotadura mandibular y el } \\
\text { margen inferior de mandíbula }\end{array}$ \\
\hline $\mathbf{V} 2$ & Ancho de rama & $\begin{array}{l}\text { Distancia sagital, perpendicular a V1, entre el punto mas anterior del margen } \\
\text { anterior de la rama mandibular y el margen posterior de la rama }\end{array}$ \\
\hline $\mathbf{V 3}$ & Altura cuerpo mandibular & $\begin{array}{l}\text { Distancia axial, paralela a V1, entre el punto cervical de la superficie posterior } \\
\text { del primer molar inferior y el margen inferior del cuerpo mandibular }\end{array}$ \\
\hline V4 & $\begin{array}{l}\text { Distancia foramen mental a línea } \\
\text { mediana }\end{array}$ & $\begin{array}{l}\text { Distancia horizontal desde el punto mas anterior del foramen mental hasta la } \\
\text { línea vertical que nace de los dos incisivos centrales inferiores }\end{array}$ \\
\hline V5 & $\begin{array}{l}\text { Distancia foramen mental y } \mathrm{m} \text { argen } \\
\text { posterior de rama mandibular }\end{array}$ & $\begin{array}{l}\text { Distancia horizontal desde el punto mas posterior del foramen mental hasta el } \\
\text { punto mas posterior de la rama mandibular }\end{array}$ \\
\hline V6 & Ancho condilar & $\begin{array}{l}\text { Distancia horizontal de los puntos de mayor distancia, mas anterior y } \mathrm{m} \text { as } \\
\text { posterior de la cabeza condilar }\end{array}$ \\
\hline V7 & Altura condilar & $\begin{array}{l}\text { Distancia vertical, perpendicular a V6, entre el punto mas superior de la } \\
\text { cabeza condilar y la base de la cabeza condilar }\end{array}$ \\
\hline V8 & Ancho cuello condilar & $\begin{array}{l}\text { Distancia horizontal, paralela a V7, entre el punto mas anterior del cuello } \\
\text { condilar y el punto mas posterior del sector posterior del cuello condilar }\end{array}$ \\
\hline V9 & $\begin{array}{l}\text { Distancia gonion a límite superior } \\
\text { del cóndilo }\end{array}$ & $\begin{array}{l}\text { Distancia axial entre el punto gonion y el punto mas superior de la cabeza del } \\
\text { cóndilo mandibular }\end{array}$ \\
\hline
\end{tabular}




\section{RESULTADOS}

La investigación se desarrolló siguiendo el protocolo antes mencionado. Se observó que para la muestra de análisis no existió diferencias significativas entre la hiperplasia condilar y el sexo de los sujetos $(\mathrm{p}<0,05)$. En la misma dirección, el lado derecho o izquierdo de la hiperplasia condilar no tuvo relación significativa con el sexo $(\mathrm{p}<0,05)$ y si presento relación significativa con la edad de los sujetos.
Las medidas realizadas se pueden observar en la Tabla II; no se presentaron valores con diferencias de medición sobre los $2 \mathrm{~mm}$ aunque si se observó diferencias significativas en las variables V1, V6, V7, V8 y V9, indicando que la $\mathrm{H}-\mathrm{HC}$ presenta mayores dimensiones en las zonas señaladas al compararla con la H-NHC.

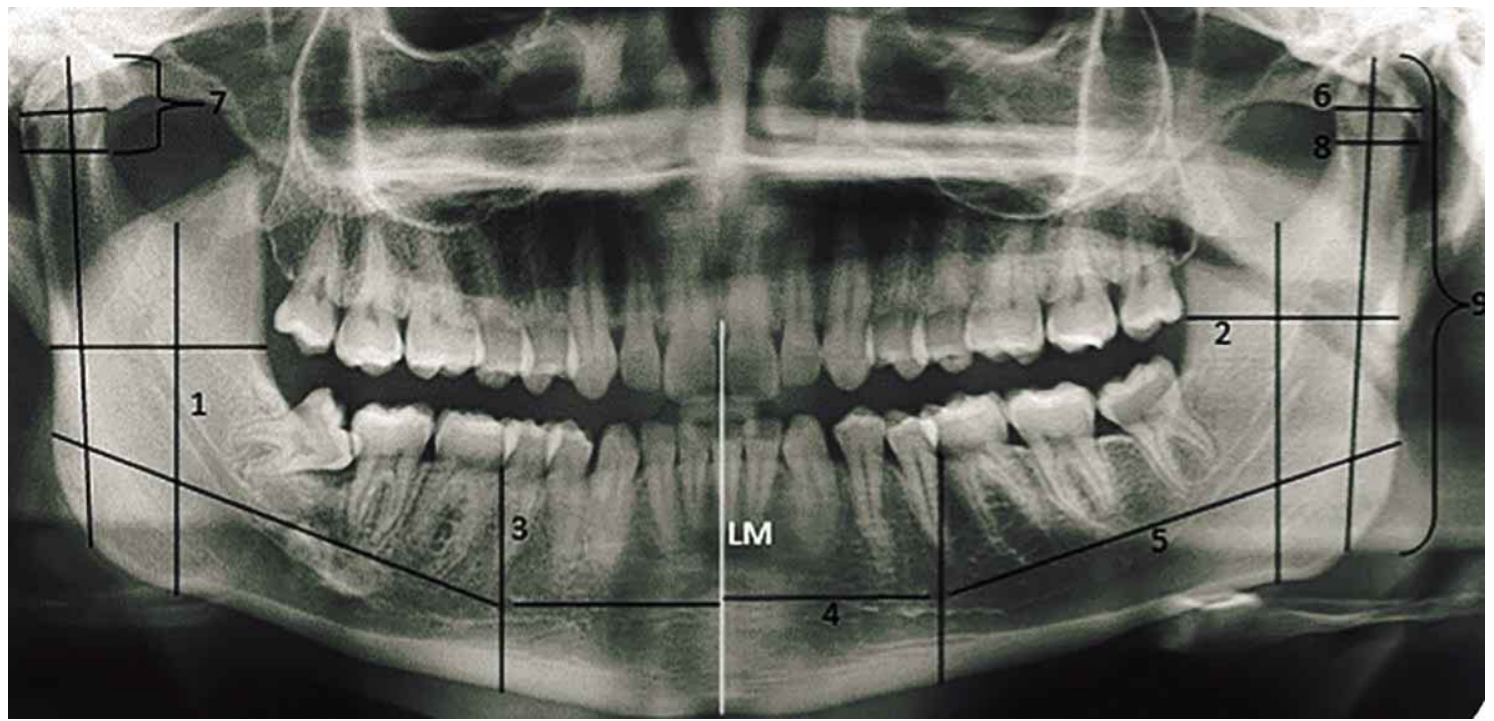

Fig. 1. Radiografía panorámica digital utilizada en esta investigación con las líneas que corresponden a las mediciones de cada una de las variables estudiadas, denominadas con numeros correlativos de acuerdo a la Tabla I.

Tabla II. Valores obtenidos en la morfometría manual realizada a las 14 radiografías de pacientes con asimetría facial asociada a hiperplasia condilar (valores en $\mathrm{mm}$ ).

\begin{tabular}{lccccccccc}
\hline Área & V1 & V2 & V3 & V4 & V5 & V6 & V7 & V8 & V9 \\
\hline HC & 48,7 & 23 & 31,2 & 25,9 & 55,4 & 8,1 & 10,3 & 8,4 & 58,6 \\
NHC & 47,7 & 23,4 & 32,2 & 25,1 & 57,4 & 8,4 & 8,6 & 8,2 & 56,2 \\
Diferencia & 1 & 0,4 & 1 & 0,8 & 2 & 0,3 & 0,3 & 0,2 & 2,4 \\
\hline
\end{tabular}

\section{DISCUSIÓN}

La metodología aplicada en este estudio se ha utilizado en otras investigaciones (Pozzer et al., 2007), y el objetivo fundamental de realizarlo fue aportar con datos relevantes para que los profesionales clínicos que generalmente cuentan con la radiografía panorámica impresa para realizar los análisis, utilizaran esta metodología para realizar parte del proceso diagnóstico de los sujetos con asimetrías faciales.

Las radiografías panorámicas son utilizadas rutinariamente en el diagnostico y evaluación de dientes y maxilares. Hoy día, la radiografía panorámica digital, de alta calidad, ha permitido el diagnóstico de calcificaciones y otras condiciones que contribuyen a un diagnóstico precoz, por ejemplo, en el análisis de placas de ateroma a nivel cervical (Garay et al., 2012, 2014).

Nuestros resultados demuestran que existen diferencias entre la H-HC y la H-NHC. No es extraño pensar en estos resultados ya que investigaciones previas han demostrado diferencias en los tamaños condilares y en la posición de los mismos (Olate et al., 2013b) cuando se 
compara la H-HC con la H-NHC. Otras investigaciones también han presentado resultados que muestran diferencias entre los tamaños condilares y mandibulares (Maeda et al., 2006).

Estos resultados señalan que existen diferencias significativas entre algunas de las mediciones realizadas; sin embargo, es importante reflexionar sobre las implicancias clínicas observadas en esas mediciones ya que diferencias de $2 \mathrm{~mm}$ o incluso de $1 \mathrm{~mm}$ fueron presentadas como significativas cuando se comparó la H-HC con la H-NHC; para los profesionales que realizan el primer diagnóstico se podría pensar que $1 \mathrm{~mm}$ de diferencia en el ancho de la rama de la hemimandíbula derecha comparada a su contralateral no son indicativos de cambios morfométricos mayores.

Es posible que la posición del paciente o los instrumentos utilizados para realizar la medición generen cambios en la toma de la imagen y en la medición de la misma (Amorim et al., 2009) de forma de que el clínico que evaluará las imágenes no tenga los elementos necesarios para realizar un correcto análisis de la radiografía. En el caso de sujetos con deformidades clase II y clase III se han identificado claras diferencias morfométricas utilizando la misma metodología (Pozzer et al.), de forma que los autores creen que la posición del paciente es muy importante en la toma de la imagen y la comparación de ambas hemimandíbulas.

Otra situación es que la limitada cantidad de unidades muestrales de nuestro estudio generen resultados clínicamente no aplicables; estas condiciones han sido observada en otras investigaciones, donde diferencias estadísticamente significativas no se vinculan con cambios clínicamente significativos (Bhardwaj et al., 2004), determinando que la valoración del clínico varíe en el momento que debe registrar valores de una enfermedad y realmente lo realize pensando solo en cambios morfológicos aceptables dentro de la normalidad (Kraemer et al., 2003).

Basado en nuestros resultados, los autores creen que cambios de menos de $2 \mathrm{~mm}$ son importantes de registrar pero también los autores creen que buena parta de los clínicos no consideraran estos valores como patológico. De esta manera, es necesario obtener y estudiar nuevas medidas y áreas que pueden ser empleadas de forma rutinaria para el diagnóstico precoz de la asimetría facial asociada a hiperplasia condilar. Cambios en tomografías computadorizadas han mostrado también variaciones de 2 $\mathrm{mm}$, considerados significativos, aunque este examen generalmente es utilizado en una segunda etapa, cuando existen reales sospechas de patología condilar (Olate et al., 2012) o cuando se encuentra en fase de planificación quirúrgica (Olate et al., 2014).

En el diagnostico de la asimetría facial y de la HC tiene un fuerte rol de la anamnesis detallada y la evolución del paciente (Raijmakers et al., 2012), lo cual hace que sean detectables por profesionales con entrenamiento; sin embargo, clínicos que no manejan regularmente estas situaciones pueden entrar en confusión cuando se enfrentan a esta patología y pueden indicar tratamientos bajo diagnósticos incorrectos (Olate \& de Moraes, 2012).

El objetivo de esta investigación fue obtener elementos diagnósticos a partir de la radiografía panorámica, pero los autores creen que la posición del paciente en la captura de la imagen, las zonas utilizadas para medir, la rigurosidad de la medición o los elementos técnicos para la medición manual pueden influir en resultados que sean clínicamente no significativos, de forma que es necesario realizar nuevas investigaciones para obtener resultados de mayor aplicación clínica.

ZAPATA, S.; MEDINA, H.; SARAVIA, D.; NAVARRO, P. \& OLATE, S. Morphometric analysis of the mandible in patients with facial asymmetry associated to condylar hyperplasia. A panoramic radiography study. Int. J. Morphol., 32(1):161-165, 2014.

SUMMARY: The aim of this research was to define the morphological characteristics in panoramic digital radiography of subjects with asymmetric facial deformities related to condylar hyperplasia. A transversal cohort study was designed for the analysis of 14 patients with this pathology, and points and lines for measurement were identified, in a comparative technique between the hemimandible with condylar hyperplasia diagnosis and hemimandible with normal growth of the condyle. In accordance with protocol all measurements were carried out manually. Variables analyzed were, size of condyle, size of the mandible ramus and size of mandible body in relation to mental foramen. The data analyses was carried out with statistical Chi-Square test considering $\mathrm{p}$ value $<0.05$ for statistical significance. The results showed some significant differences between both sides of the mandible in five of nine evaluations. However, measurement with less than $2 \mathrm{~mm}$ differences were obtained, which clinically might not be relevant, despite statistical differences. In conclusion, new measurements in others areas or with different methodologies are necessary to better determine clinical and statistical differences for early diagnosis of condylar hyperplasia.

KEY WORDS: Facial assymetry; Panoramic radiography; Condylar hiperplasia. 


\section{REFERENCIAS BIBLIOGRÁFICAS}

Amorim, M.M.; Borini, C.B.; Lopes, S.L.P.C.; Haiter-Neto, F. \& Caria, P.H.F. Morphological description of mandibular canal in panoramic radiographs of Brazilian subjects: Association between anatomic characteristic and clinical procedures. Int. J. Morphol., 27(4):1243-8, 2009.

Bhardwaj, S.; Camacho, F.; Derrow, A.; Fleischer, A. \& Feldman, S. Statistical significance and clinical relevance. The importance of power in clinical trials in dermatology. Arch. Dermatol., 140(12):1520-3, 2004.

Dryland, K. \& Ellis, E. Diagnosis and treatment planning for the surgical-orthodontic patient. Dent. Clin. North Am., 34(2):36184, 1990.

Ferrario, V. F.; Sforza, C.; Poggio, C. E. \& Tartaglia, G. Distance from symmetry: A three dimensional evaluation of facial asymmetry. J. Oral Maxillofac. Surg., 52(11):1126-32, 1994.

Garay, I. \& Olate, S. Consideraciones actuales en el estudio imagenológico de las calcifiaciones de tejidos blandos en zona de ángulo mandibular. Int. J. Odontostomat., 7(3):455-64, 2013

Garay, I.; Netto, H., D. \& Olate S. Soft tissue calcified in mandibular angle area observed by means of panoramic radiography. Int. J. Clin. Exp. Med., 7(1):51-6, 2014.

Kraemer, H. C.; Morgan, G. A.; Leech, N. L.; Gliner, J. A.; Vaske, J. J.\& Harmon, R. J. Measures of clinical significance. J. Am. Acad. Child Adolesc. Psychiatry, 42(12):1524-9, 2003.

Maeda, M.; Katsumata, A.; Ariji, Y.; Muramatsu, A.; Yoshida, K.; Goto, S.; Kurita, K. \& Ariji, E. 3D-CT evaluation of facial asymmetry in patients with maxillofacial deformities. Oral Surg. Oral Med. Oral Pathol. Oral Radiol. Endod., 102(3):38290, 2006.

Olate, S. \& de Moraes, M. Asymmetric Facial Deformity. Role of CondylarHyperplasia. Int. J. Odontostomat., 6(3):337-47, 2012.

Olate, S.; Almeida, A.; Alister, J. P.; Navarro, P.; Netto, H. D.; de Moraes, M. Facial asymmetry and condylar hyperplasia: considerations for diagnosis in 27 consecutive patients. Int. J. Clin. Exp. Med., 6(10):937-41, 2013a.

Olate, S.; Cantín, M.; Alister, J.P.; Uribe, F.; Navarro, P.; Olate, G. \& de Moraes, M. Relationship Between Condylar Size and Transverse Facial Asymmetry in Subject with Condylar Hyperplasia. Int. J. Morphol., 31(3):937-41, 2013 b.

Olate, S.; Netto, H., D.; Rodriguez-Chessa, J.; Alister, J. P.; de Albergaria-Barbosa, J. \& de Moraes, M. Mandible condylar hyperplasia: a review of diagnosis and treatment protocol. Int. J. Clin. Exp. Med., 6(9):727-37, 2013c.
Olate, S.; Unibazo, A.; Almeida, A. \& de Moraes, M. Mandibular condylectomy revisited: Technical notes concerning the use of an ultrasonic system. J. Oral Maxillofac. Surg., 72(3):4814, 2014.

de Oliveira, M.; de Moraes, P. H.; Olate, S.; Alonso, B. C.; Watanabe, P. C. A.; Haiter-Neto, F. \& Albergaria-Barbosa, J.R. Morphometric study of mandibular ramus related to sagittal ramus split osteotomy and osteosynthesis. J. Craniofac. Surg., 23(5):1484-7, 2012.

Pozzer, L.; Olate, S.; Asprino, L. \& de Moraes, M. ¿Existen diferencias en la morfometría mandibular de pacientes candidatos a cirugía ortognática?. Parte 1: Influencias de la clase facial. Int. J. Morphol., 27(3):751-6, 2009.

Raijmakers, P. G.; Karssemakers, L. H. \& Tuinzing, D. B. Female predominance and effect of gender on unilateral condylar hiperplasia: a review and meta-analysis. J. Oral Maxillofac. Surg., 70(1):e72-6, 2012.

\author{
Dirección para Correspondencia: \\ Prof. Dr. Sergio Olate \\ División de Cirugía Oral y Maxilofacial \\ Universidad de La Frontera \\ Claro Solar 115, Oficina 414-A \\ Temuco \\ CHILE
}

Email: sergio.olate@ufrontera.cl

Recibido : 11-09-2013

Aceptado: 27-12-2013 